The Astrophysical Journal, 287:L99-L103, 1984 December 15

(C) 1984. The American Astronomical Society. All rights reserved. Printed in U.S.A.

\title{
EVIDENCE THAT THE ANOMALOUS COSMIC-RAY COMPONENT IS SINGLY IONIZED
}

\author{
A. C. Cummings and E. C. Stone \\ California Institute of Technology \\ AND \\ W. R. WEBBER \\ University of New Hampshire \\ Received 1984 July 2; accepted 1984 September 24
}

\begin{abstract}
We have used data from the Cosmic Ray Subsystem on the Voyager spacecraft to measure the spectra of He, $\mathrm{C}, \mathrm{N}, \mathrm{O}$, and Ne with 4-124 MeV per nucleon (for O) during solar minimum conditions near the end of 1977 . By subtracting both a low-energy interplanetary component and the high-energy galactic component we have determined the energy spectra of the "anomalous" cosmic-ray species $\mathrm{He}, \mathrm{N}, \mathrm{O}$, and $\mathrm{Ne}$. The energy dependences of such spectra depend upon the charge state of the particles, their source spectra, and the rigidity dependence of the diffusion coefficient. If the source spectra at the boundary of the modulation region have similar power-law energy dependences, then the observed energy spectra indicate that the anomalous particles are singly ionized.
\end{abstract}

Subject heading: cosmic rays: general

\section{INTRODUCTION}

The anomalous component of low-energy cosmic rays consists mainly of $\mathrm{He}, \mathrm{N}, \mathrm{O}$, and $\mathrm{Ne}$, an unusual composition that presents several intriguing questions concerning its origin. It has been suggested that the anomalous particles might originate from a galactic source and are presumably either partly or fully ionized (McDonald et al. 1974, 1977; Hoyle and Clayton 1974; Biswas, Durgaprasad, and Trevedi 1981). However, this would require significant modification of cosmic-ray modulation theory (Fisk 1976). An alternative suggestion is that they are singly charged particles accelerated in the heliosphere or at its boundary from local interstellar neutrals ionized in the solar system (Fisk, Kozlovsky, and Ramaty 1974; Pesses, Jokipii, and Eichler 1981), thus providing a natural explanation for the observation that only particles with first ionization potentials $\geq 13.6 \mathrm{eV}$ are anomalous. Although indirect evidence that the anomalous particles are singly ionized has been presented (McKibben 1977; Klecker et al. 1980), these arguments have been disputed (Paizis and von Rosenvinge 1981). In this Letter we have adopted an approach which utilizes the differences in the energy spectra of the anomalous $\mathrm{He}, \mathrm{N}, \mathrm{O}$, and $\mathrm{Ne}$ to gain additional information on the charge state of the particles. A preliminary account of this work has been reported elsewhere (Webber, Cummings, and Stone 1983).

\section{DATA ANALYSIS}

In this analysis individual spectra are obtained with the Cosmic Ray Subsystem (CRS) (Stone et al. 1977) for He, C, $\mathrm{N}, \mathrm{O}$, and Ne nuclei for a 90 day quiet period between 1977 September and 1978 February. During this time period the Voyager spacecraft were between $\sim 1.3$ and $2.5 \mathrm{AU}$ from the
Sun, and there was minimum modulation of the cosmic-ray fluxes. The quiet-time intervals were selected such that the 4.5-7.8 MeV per nucleon $\mathrm{He}$ intensity was typically $<0.7$ particles $\left(\mathrm{m}^{2} \mathrm{~s} \text { sr } \mathrm{MeV} \text { per nucleon }\right)^{-1}$.

The observed spectra for $\mathrm{He}, \mathrm{C}, \mathrm{N}, \mathrm{O}$, and $\mathrm{Ne}$ are shown in Figure 1. A turn-up exists in the low-energy $\mathrm{He}$ and $\mathrm{C}$ spectra, probably due to a low-level solar or interplanetary component which was not eliminated by the stringent quiet-time selection criterion. The solid lines in Figures $1 b, 1 c, 1 d$, and $1 e$ are scaled from the He spectrum of this component assuming a charge composition similar to that for corotating events (He:C:N:O:Ne = 16500:100:15:95:19) (from Gloeckler et al. 1979 and assuming $\mathrm{N} / \mathrm{O}=0.13$ ). Using this scaling, or a similar scaling based on solar flare abundances, there is excellent agreement with the observed $\mathrm{C}$ spectrum, suggesting that the low-energy turn-up is of solar/interplanetary origin with no evidence of an anomalous $\mathrm{C}$ component. The dashed lines in Figure 1 show the estimated spectra of galactic cosmic rays scaled from the observed high-energy $\mathrm{C}$ spectrum with a normalization of He:C:N:O:Ne = 5000:100:25:91:11 (similar to ratios in Garcia-Munoz and Simpson 1979).

\section{DISCUSSION}

The spectrum of anomalous He shows a broad maximum in the $10-20 \mathrm{MeV}$ per nucleon region. The anomalous $\mathrm{N}$ and $\mathrm{O}$ spectra have regions with small slope, suggestive of a peak in their spectra near $5 \mathrm{MeV}$ per nucleon. Although the $\mathrm{Ne}$ spectrum is statistically less well determined, there is no evidence for a peak down to $4 \mathrm{MeV}$ per nucleon. We will now show how these differences in peak locations provide information on the origin and charge state of the anomalous component. 

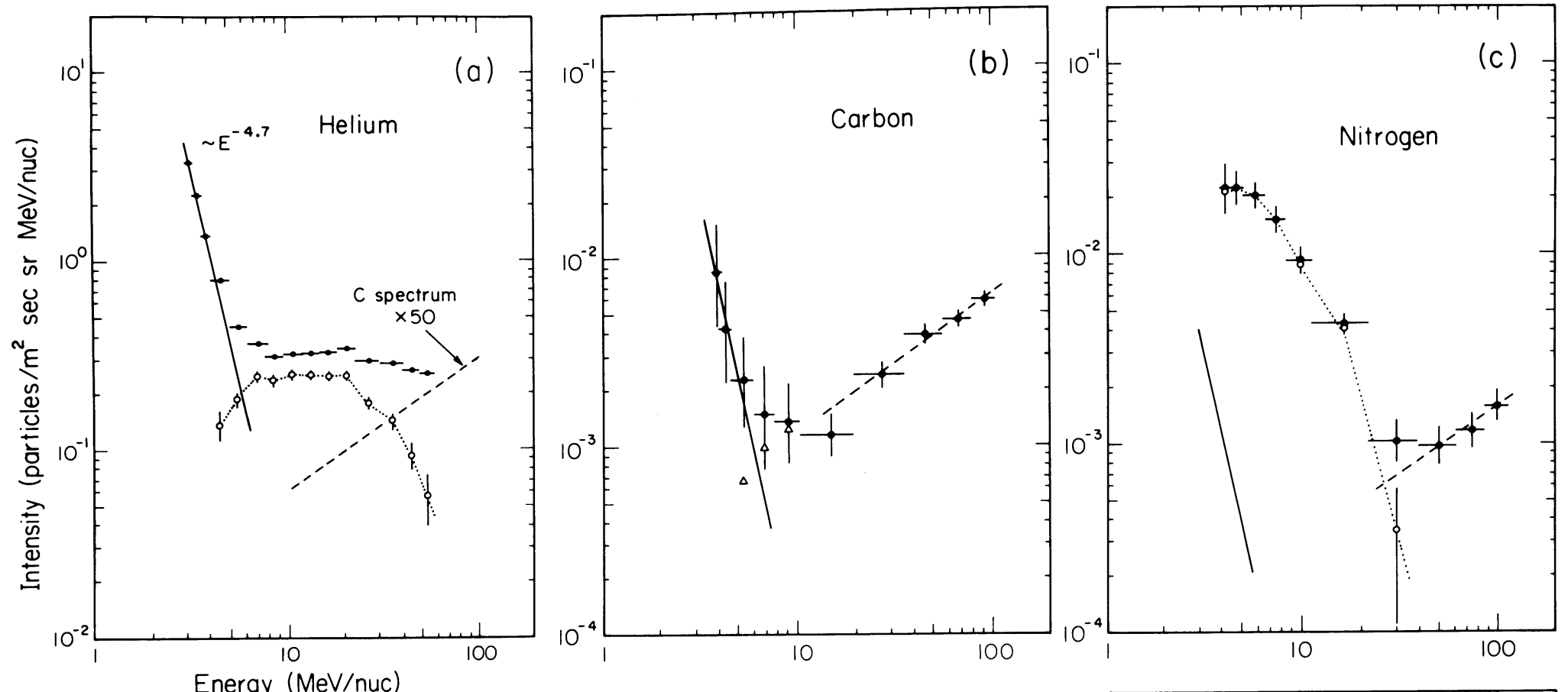

FIG. 1.-Quiet-time energy spectra of helium $(a)$, carbon $(b)$, nitrogen $(c)$, oxygen $(d)$, and neon $(e)$ from Voyager 1 and Voyager 2 for 1977 September-1978 February. The observed data are shown as solid circles. Estimated spectra of solar/interplanetary and galactic components are shown as solid and dashed lines, compor respectively. In (a), (c), (d), and (e) the spectra of anomalous cosmic rays are indicated by $O$ 's which are joined by the dotted lines. In $(b)$ the $\Delta$ 's are obtained by subtraction of solid line from data.
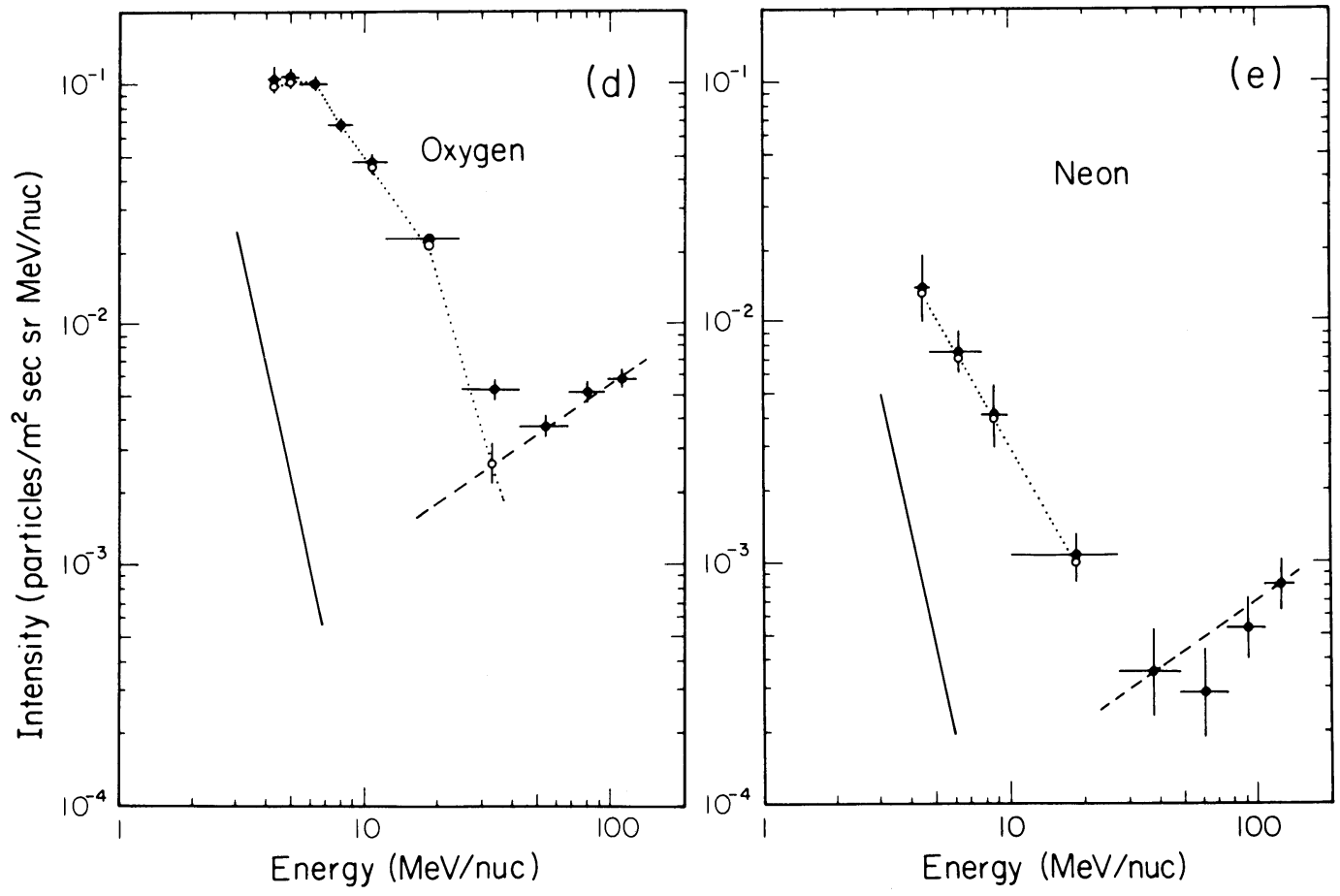
Cosmic-ray spectra observed near Earth, such as those shown in Figure 1, are determined both by the spectra at the boundary of the modulation region and by interplanetary propagation conditions. Recently, Fisk (1983) has suggested that if the input spectra at the modulation boundary are similar, then characteristic spectral features observed near Earth, such as spectral peaks, should occur at an energy for each species for which the particles have the same interplanetary diffusion coefficient $\kappa$. If we use the conventional assumption that $\kappa \propto \beta R^{\gamma}$, where $\beta$ is the particle velocity and $R$ is rigidity, then for nonrelativistic particles, with energy $E$ in $\mathrm{MeV}$ per nucleon, $R \propto A E^{1 / 2} / Z$ and $\beta \propto E^{1 / 2}$, so that $\kappa \propto$ $(A / Z)^{\gamma} E^{(\gamma+1) / 2}$. Thus for a given value of $\kappa$, the corresponding particle energies scale as

$$
f_{E}(Z, A) \propto(A / Z)^{-2 \gamma /(\gamma+1)} .
$$

For fully ionized particles with $A / Z=2$, the energy scaling factor $f_{E}$ is independent of $Z$ and $\gamma$, and the location of the peaks in the spectra should occur at the same value of energy per nucleon, as is observed for several species of galactic cosmic-ray nuclei such as $\mathrm{C}, \mathrm{O}, \mathrm{Ne}, \mathrm{Mg}$, and $\mathrm{Si}$ (see, e.g., Garcia-Munoz et al. 1977). For singly ionized particles ( $Z=1$ in eq. [1]),

$$
f_{E} \propto A^{-2 \gamma /(\gamma+1)},
$$

and the spectra should peak at different energies for particles with different $A$, as is observed for the anomalous species shown in Figures $1 a, 1 c, 1 d$, and $1 e$ (see also Klecker 1977).

The spectra of Figure 1 do not show well-defined peaks for which an energy scaling factor can be accurately derived. However, using numerical solutions (based on the method described by Fisk 1971) to the conventional cosmic-ray transport equation (including the effects of diffusion, convection, and adiabatic deceleration), we find that for boundary spectra which are power laws in kinetic energy with the same index, not only do the peak energies of the modulated spectra scale as indicated by equation (1), but the entire spectra scale as well. Figure 2 shows an example of this scaling in which a boundary $(=50 \mathrm{AU})$ spectrum proportional to $E^{-5}$, solar wind velocity $=400 \mathrm{~km} \mathrm{~s}^{-1}$, and a diffusion coefficient $=$ $\kappa_{0} \beta R^{0.8}$ (in $\mathrm{cm}^{2} \mathrm{~s}^{-1}$ for $\kappa_{0}=2.4 \times 10^{22}$ and $R$ in GV) have been used in the numerical study. The two calculated spectra for $1 \mathrm{AU}$ are for singly charged $\mathrm{O}$ and $\mathrm{He}$, which can be seen to coincide when appropriately scaled. The choices of $\kappa_{0}$ and the $E^{-5}$ spectrum are not unique; other power-law spectra and/or other $\kappa_{0}$ 's give the same energy scaling factor but different intensity factors. This result suggests that the measured spectrum of He when scaled in energy by an appropriate factor $\left(f_{E}\right)$ would have the same energy dependence as that of $O$. (For a similar argument in the case of simple diffusion, see Klecker et al. 1977.) Although the observed energy dependence may differ from that shown in Figure 2, for example, because of nonspherical symmetry of the source or a different rigidity dependence of the diffusion coefficient, we would expect all anomalous species to be similarly affected and the spectra to still be related by the energy scaling factor in equation (1).

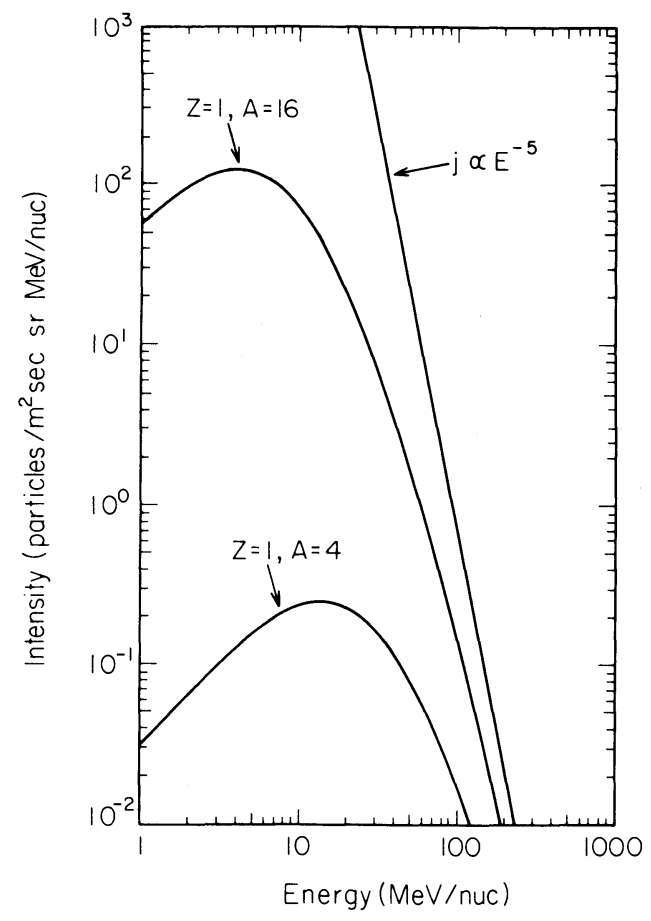

FIG. 2.-Calculated spectra at $1 \mathrm{AU}$ of singly charged oxygen and helium shown with an assumed boundary spectrum. The calculated spectra coincide when the helium spectrum is shifted in energy by the factor 0.292 (from eq. [2] with $A=16 / 4=4$ and $\gamma=0.8$ ) and in intensity by the factor 494 .

In order to empirically determine these energy scaling factors for the anomalous component, we used the anomalous $\mathrm{O}$ spectrum as the reference spectrum (dotted line in Fig. 1d) and scaled the other spectra in energy and intensity to obtain the best fit in the regions of overlap. The resulting spectra of $\mathrm{He}, \mathrm{N}$, and $\mathrm{Ne}$ scaled to $\mathrm{O}$ are shown along with the $\mathrm{O}$ spectrum in Figure 3. The values of the energy scaling factors which minimized the $\chi^{2}$ of the fit between the spectra of the different species are shown in Figure 4. The $1 \sigma$ uncertainties correspond to values of $f_{E}(Z, A)$ which gave $\chi^{2}=\left(\chi^{2}\right)_{\min }+$ 1. The error bar on the $O$ point is the result of fitting the $O$ spectrum to its power-law segmented estimate and indicates the level of systematic uncertainty thus introduced. The solid lines in Figure 4 show the dependence of $f_{E}$ on $A$ for various values of $\gamma$ (in $\kappa \propto \beta R^{\gamma}$ ), assuming $Z=1$. The dashed line at $f_{E}=1$ indicates the mass-independent scaling factor for fully ionized particles.

It is clear from Figure 4 that the observed mass dependence of $f_{E}$ is inconsistent with an assumption that the particles are fully ionized. On the other hand, if the particles are assumed to be singly ionized then the mass dependence of the scaling factor is consistent with $\gamma \approx 0.7-0.9$. This value of $\gamma$ is consistent with observational evidence and theoretical predictions of the behavior of the diffusion coefficient in the rigidity range $(0.4-4 \mathrm{GV})$ appropriate for singly ionized $\mathrm{He}$ and $\mathrm{O}$ with 5-30 MeV per nucleon (see, e.g., Palmer 1982).

If this approach is correct then similar considerations should apply to the modulation of galactic cosmic rays of different $A / Z$. For example, the peak in the spectrum of hydrogen 


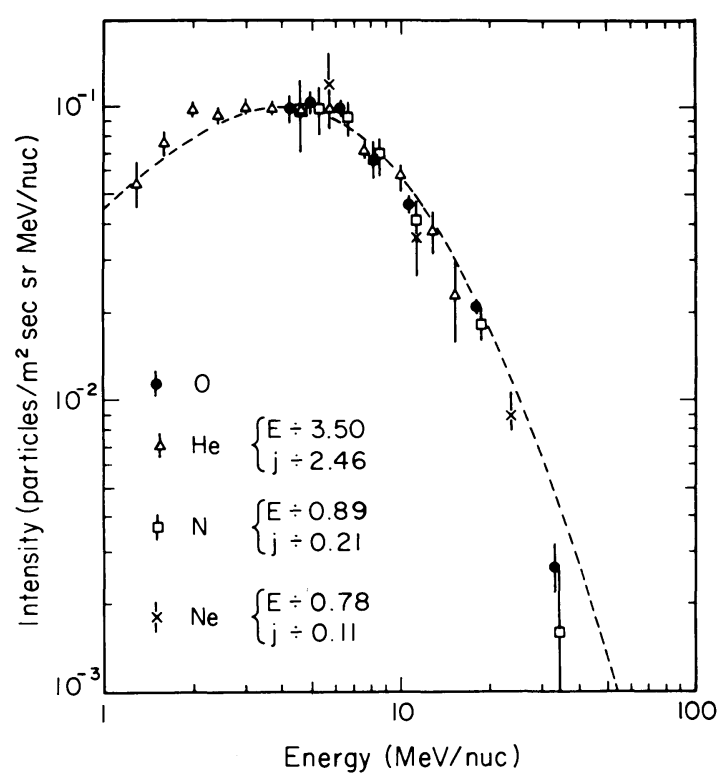

FIg. 3.-Energy spectra of helium, nitrogen, and neon scaled to the oxygen spectrum with the best fit factors in energy and intensity as described in the text. Shown for reference (dashed line) is the calculated spectrum of singly charged oxygen from Fig. 2 (normalized to a peak intensity of 0.1 particles $\left[\mathrm{m}^{2} \mathrm{~s} \text { sr MeV per nucleon }\right]^{-1}$ ).

( $A / Z=1$ ) would be expected to occur at an energy which is a factor of $\sim 1.85$ larger than the corresponding energy per nucleon peak of the helium $(A / Z=2)$ spectrum (for $\gamma=0.8$ ) if the unmodulated spectra were power laws in kinetic energy of the same index. Measurements of these spectra at approximately the same time in 1977 (Evenson et al. 1983; Webber and Yushak 1983) yield a ratio of $\sim 1.5$. This small difference indicates that the unmodulated spectra of the galactic component are probably not simple power laws in kinetic energy. However, by using unmodulated spectra of galactic $\mathrm{He}$ and $\mathrm{H}$ of the form $(E+400)^{-2.65}$ and by using a galactic electron spectrum derived from the interstellar radio data, reasonable fits can be made to the 1977 spectra of $\mathrm{H}$, $\mathrm{He}$, and electrons at Earth using the same diffusion coefficient as in the anomalous component study.

It is possible, of course, to produce arbitrary peak locations for the anomalous cosmic-ray particles, whether singly or fully ionized, if the unmodulated spectra are not constrained to be similar for the different charges. For example, for the same diffusion coefficient discussed earlier, our numerical studies indicate that an unmodulated spectrum of fully ionized $\mathrm{O}$ proportional to $E^{-16}$ and of fully ionized He proportional to $E^{-8}$ could account for the observed near-Earth spectra. However, as pointed out by Fisk (1976), the implied interstellar

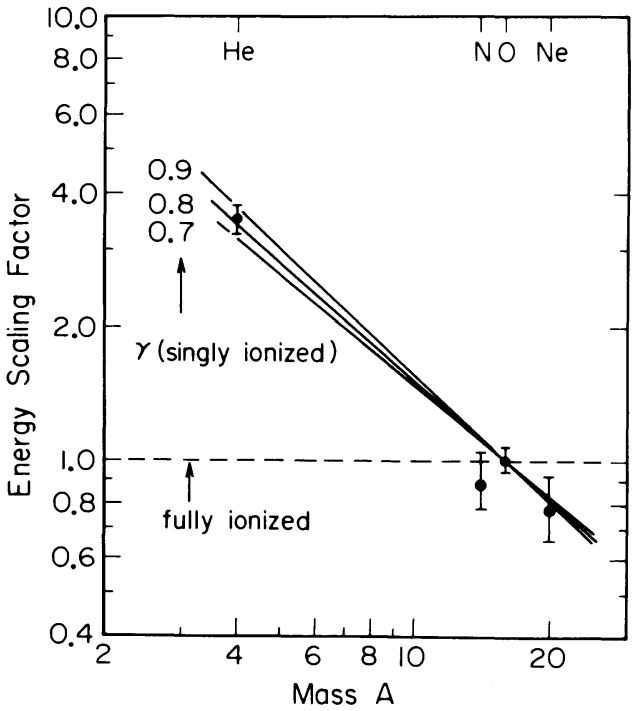

FIG. 4.-Energy scaling factor $f_{E}$ vs mass $A$. Determination of the data points is described in the text. The solid lines show the expected dependence on mass for singly ionized particles for three values of $\gamma$ (in $\kappa \propto \beta R^{\gamma}$ ). The dashed line at $f_{E}=1$ indicates the expected value for fully ionized particles with $A / Z=2$ which is independent of $\gamma$.

fluxes are unreasonably large, exceeding by many orders of magnitude those allowed from arguments based on the energy density of cosmic rays and the ionization state of trace elements in the interstellar medium. Even if fully ionized particles were not interstellar but were locally accelerated, the production of such spectra would require additional ad hoc assumptions. We are suggesting, on the other hand, that a simple model, in which the anomalous component is singly ionized and the solar modulation is represented by a conventional transport model with a realistic diffusion coefficient, can account for the observed spectral features of both the galactic and anomalous components when reasonable forms of the unmodulated spectra are assumed.

We are grateful to R. E. Vogt for his contributions as Principal Investigator for the Cosmic Ray Subsystem on the Voyager spacecraft. We also appreciate the contributions of the other CRS team members consisting of scientists and engineers at the California Institute of Technology, the Goddard Space Flight Center, the University of Arizona, and the University of New Hampshire. We also thank R. A. Mewaldt for several useful discussions. This work was supported in part by NASA under contract NAS 7-918 and grants NAGW-200 and NGR 05-002-160.

\section{REFERENCES}

Biswas, S., Durgaprasad, N., and Trevedi, Sushma S. 1981, Earth Planet. Sci., 90, 337.

Evenson, P., Garcia-Munoz, M., Meyer, P., Pyle, K. R., and Simpson, J. A. 1983, Ap. J. (Letters), 275, L15.
Fisk, L. A. 1971, J. Geophys. Res., 76, 221.

1976, Ap. J., 206, 333.

1983, private communication. 
Fisk, L. A., Kozlovsky, B., and Ramaty, R. 1974, Ap. J. (Letters), 190, L35.

Garcia-Munoz, M., Mason, G. M., Simpson, J. A., and Wefel, J. P. 1977, Proc. 15th Int. Cosmic Ray Conf. (Plovdiv), 1, 230.

Garcia-Munoz, M., and Simpson, J. A. 1979, Proc. 16th Int. Cosmic Ray Conf. (Kyoto), 1, 270.

Gloeckler, G., Hovestadt, D., Ipavich, F. M., and Mason, G. M. 1979, Proc. 16th Int. Cosmic Ray Conf. (Kyoto), 5, 368.

Hoyle, F., and Clayton, D. D. 1974, Ap. J., 191, 705.

Klecker, B. 1977, J. Geophys. Res., 82, 5287.

Klecker, B., Hovestadt, D., Gloeckler, G., and Fan, C. Y. 1977, Ap. J., 212, 290

1980, Geophys. Res. Letters, 7, 1033

McDonald, F. B., Lal, N., Trainor, J. H., Van Hollebeke, M. A. I., and Webber, W. R. 1977, Ap. J., 216, 930.
McDonald, F. B., Teegarden, B. J., Trainor, J. H., and Webber, W. R. 1974, Ap. J. (Letters), 187, L105.

McKibben, R. B. 1977, Ap. J. (Letters), 217, L113.

Paizis, C., and von Rosenvinge, T. T. 1981, Proc. 17th Int. Cosmic Ray Conf. (Paris), 10, 73.

Palmer, I. D. 1982, Rev. Geophys. Space Phys., 20, 335.

Pesses, M. E., Jokipii, J. R., and Eichler, D. 1981, Ap. J. (Letters), 246, L85.

Stone, E. C., Vogt, R. E., McDonald, F. B., Teegarden, B. J., Trainor, J. H., Jokipii, J. R., and Webber, W. R. 1977, Space Sci. Rev., 21, 355.

Webber, W. R., Cummings, A. C., and Stone, E. C. 1983, Proc. 18th Int. Cosmic Ray Conf. (Bangalore), 9, 95.

Webber, W. R., and Yushak, S. M. 1983, Ap. J., 275, 391.

A. C. Cummings: 220-47 Downs Laboratory, California Institute of Technology, Pasadena, CA 91125

E. C. Stone: 103-33 E. Bridge, California Institute of Technology, Pasadena, CA 91125

W. R. WebBeR: Space Sciences Center, DeMeritt Hall, University of New Hampshire, Durham, NH 03824 